\title{
Exploring the potentials and limitations of the time-reversal imaging of finite seismic sources
}

\author{
S. Kremers ${ }^{1}$, A. Fichtner ${ }^{1, *}$, G. B. Brietzke ${ }^{1, * *}$, H. Igel ${ }^{1}$, C. Larmat ${ }^{2}$, L. Huang ${ }^{2}$, and M. Käser ${ }^{1}$ \\ ${ }^{1}$ Department of Earth and Environmental Sciences, Ludwig-Maximilians-University, Theresienstr. 41/III, \\ 80333 Munich, Germany \\ ${ }^{2}$ EES-17 Geophysics Group, Los Alamos National Laboratory, Los Alamos, NM 87545, USA \\ *now at: Faculty of Geosciences, Utrecht University, Utrecht, The Netherlands \\ ***now at: GFZ German Research Centre for Geosciences, Potsdam, Germany
}

Received: 28 January 2011 - Published in Solid Earth Discuss.: 17 March 2011

Revised: 30 May 2011 - Accepted: 31 May 2011 - Published: 21 June 2011

\begin{abstract}
The characterisation of seismic sources with timereversed wave fields is developing into a standard technique that has already been successful in numerous applications. While the time-reversal imaging of effective point sources is now well-understood, little work has been done to extend this technique to the study of finite rupture processes. This is despite the pronounced non-uniqueness in classic finite source inversions.

The need to better constrain the details of finite rupture processes motivates the series of synthetic and real-data time reversal experiments described in this paper. We address questions concerning the quality of focussing in the source area, the localisation of the fault plane, the estimation of the slip distribution and the source complexity up to which timereversal imaging can be applied successfully. The frequency band for the synthetic experiments is chosen such that it is comparable to the band usually employed for finite source inversion.

Contrary to our expectations, we find that time-reversal imaging is useful only for effective point sources, where it yields good estimates of both the source location and the origin time. In the case of finite sources, however, the timereversed field does not provide meaningful characterisations of the fault location and the rupture process. This result cannot be improved sufficiently with the help of different imaging fields, realistic modifications of the receiver geometry or weights applied to the time-reversed sources.

The reasons for this failure are manifold. They include the choice of the frequency band, the incomplete recording of wave field information at the surface, the excitation of large-
\end{abstract}

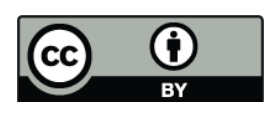

Correspondence to: S. Kremers (kremers@geophysik.uni-muenchen.de) amplitude surface waves that deteriorate the depth resolution, the absence of a sink that should absorb energy radiated during the later stages of the rupture process, the invisibility of small slip and the neglect of prior information concerning the fault geometry and the inherent smoothness of seismologically inferred Earth models that prevents the beneficial occurrence of strong multiple-scattering.

The condensed conclusion of our study is that the limitations of time-reversal imaging - at least in the frequency band considered here - start where the seismic source stops being effectively point-localised.

\section{Introduction}

Time reversal (TR) is a universal concept that can be found in numerous physical sciences, including meteorology (e.g. Talagrand and Courtier, 2007), geodynamics (e.g. Bunge et al., 2003), ground water modelling (e.g. Sun, 1994) and seismology. The misfit $\chi$ between observed and synthetic data is propagated backwards in time to detect the underlying discrepancies between the real world and its mathematical model. TR can be approached from two closely related directions: (1) the invariance of a non-dissipative physical system with respect to a sign change of the time variable, and (2) the computation of the gradient of $\chi$ with the help of the adjoint method.

From a seismological perspective, the time-invariance of perfectly elastic wave propagation provides the intuitive justification for the TR imaging of seismic sources: Seismograms $\boldsymbol{u}_{0}\left(\boldsymbol{x}^{r}, t\right)$ recorded at positions $\boldsymbol{x}^{r}(r=1, \ldots, n)$ are reversed in time, re-injected as sources at their respective receiver locations and the resulting wave field $\boldsymbol{u}(\boldsymbol{x}, t)$ is then

Published by Copernicus Publications on behalf of the European Geosciences Union. 
propagated backwards in time through an appropriate Earth model. When the receiver configuration is sufficiently dense, the time-reversed wave field $\boldsymbol{u}$ approximates the original wave field $\boldsymbol{u}_{0}$. Focussing of $\boldsymbol{u}$ then occurs at the time and location where $\boldsymbol{u}$ was excited, thus, providing information on the original earthquake source.

While being mathematically more rigorous, the adjoint method (e.g. Tarantola, 1988; Tromp et al., 2004; Fichtner et al., 2006; Fichtner, 2010) leads to a similar result: The gradient of the misfit $\chi$ with respect to the source parameters is given in terms of the time-reversed wave field generated by adjoint sources that radiate the misfit from the receiver positions back into the Earth model. In the case of a moment tensor point source, for instance, the derivative of $\chi$, with respect to the moment tensor $\boldsymbol{M}$, is given by

$$
\frac{\partial \chi}{\partial M_{i j}}=-\int \epsilon_{i j}\left(\boldsymbol{x}^{s}, t\right) d t,
$$

where $\epsilon_{i j}$ and $\boldsymbol{x}^{s}$ denote the strain tensor computed from the time-reverse field $\boldsymbol{u}$ and the source position, respectively. In this sense, TR can be interpreted as the first step in an iterative gradient-based source inversion (e.g. Tromp et al., 2004; Hjörleifsdóttir, 2007; Fichtner, 2010).

The history of TR imaging is likely to have started in ocean acoustics (e.g. Parvulescu and Clay, 1965; Derode et al., 1995; Edelmann et al., 2002), from where it migrated to medical imaging (e.g. Fink, 1997; Fink and Tanter, 2010), nondestructive testing (e.g. Chakroun et al., 1995; Sutin et al., 2004) and many other fields. One of the earliest seismic applications can be found in the work of McMechan (1982) who introduced TR source imaging as a modified version of migration. The time-reversed wave equation is used to image earthquake sources instead of subsurface structures (Artman et al., 2010). Kennett (1983) pinpointed the advantages of TR as early as 1983: (1) no prior interpretation of the timeseries is needed and (2) the full elastic wave field is used to obtain the best image of the source. Early applications were limited to structurally simple or acoustic models (e.g. McMechan et al., 1985; Rietbrock and Scherbaum, 1994; Fink, 1996), but recent advances in numerical modelling enabled applications in more complex scenarios with different types of seismic sources, including the classic double couple point source (Gajewski and Tessmer, 2005), extended faults (Ishii et al., 2005; Larmat et al., 2006; Allmann and Shearer, 2007), micro-seismic tremor (Steiner et al., 2008) and volcanic long-period events (O'Brien et al., 2011). Larmat et al. (2009) demonstrate the need to use specific imaging fields such as divergence or strain to distinguish sources from low velocity zones.

While TR imaging of effective point sources is now wellunderstood, little has been done to explore its potential to detect the details of finite rupture processes. This is surprising because classical finite-source inversions (e.g. Cotton and Campillo, 1995; Cesca et al., 2010) are known to be highly non-unique (Mai et al., 2007). The urgent need to improve finite-source inversions motivates this study where we attempt to answer several key questions with the help of both synthetic and real-data experiments: (1) How well does the time-reversed field focus in the source area? (2) Does TR imaging provide constraints on the source volume? (3) Can regions with large slip (asperities) be identified? (4) Can the rupture speed be estimated? (5) Up to which level of complexity does TR imaging provide useful information on the rupture process?

This paper is organised as follows: In a first series of synthetic tests, we study TR imaging of single and multiple point sources under nearly ideal conditions. We then extend our experiments to synthetic data computed from a finite-rupture model. To improve the focussing of the time-reversed field, we investigate the influence of the station configuration and the weighting of the adjoint sources. Finally, we provide an application to the strong-motion data recorded during the 2000 Tottori (Japan) earthquake.

\section{Numerical method}

For our TR experiments, we employ a spectral-element algorithm to model wave propagation in 3-D elastic media (Fichtner and Igel, 2008; Fichtner et al., 2009a,b). The model volume is divided into equal-sized hexahedral elements, and Perfectly Matched Layers (PML) are used to avoid reflections from the nonphysical model boundaries. In the interest of simplicity, we restrict ourselves to isotropic and nondissipative media.

The model used in our synthetic tests is $160 \times 170 \times 40 \mathrm{~km}$ wide. It comprises $60 \times 60 \times 16$ elements, which corresponds to $\sim 3$ million grid points when the polynomial degree is 4 . This setup allows us to model wave fields with frequencies up to $2 \mathrm{~Hz}$. Both the receiver configuration (Fig. 1, left) and the structural model (Fig. 1, right) in most of our simulations are the same as in the SPICE source inversion benchmark (Mai et al., 2007) that was intended to mimic the circumstances of the 2000 Tottori (Japan) earthquake. For the real data experiment, we use the Japanese KiK-net stations (Fig. 11) and the layered velocity model of Semmane (2005). As we intend to work in the frequency range of kinematic source inversions $(f=0.1-1 \mathrm{~Hz})$ the velocity models were chosen alike. Even if the models seem dramatically smooth for time-reversal purposes, we argue that no unknown complexity should be added.

To generate the time-reversed wave field, the displacement is recorded at the surface receivers, flipped in time and then re-injected as three-component adjoint sources. For the propagation of the reverse field we use the same algorithm, setup and velocity model as for the forward simulation. 


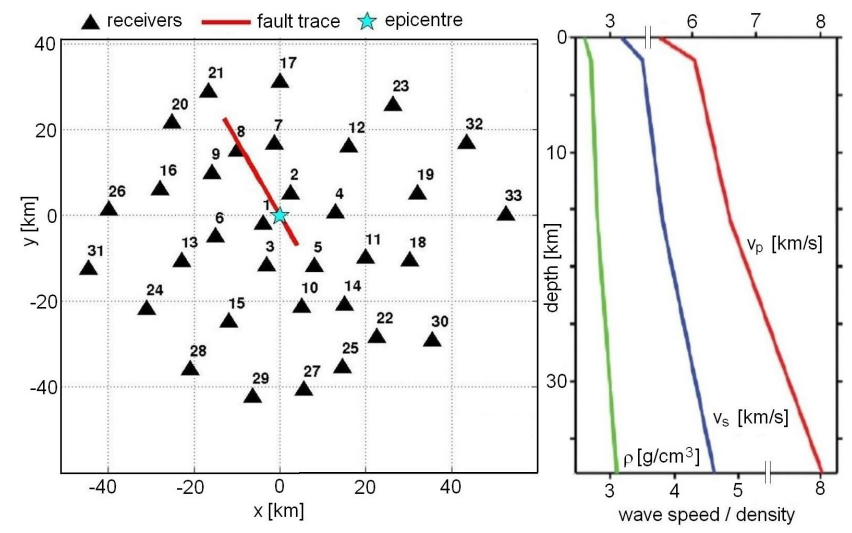

Fig. 1. Left: Geographic model setup. Stations are marked by triangles. The red line and the star mark the fault trace and the epicentre for the finite-fault simulations in Sect. 4. Right: Velocity and density model used in all synthetic simulations.

\section{Synthetic points source simulations}

\subsection{Single point source}

Our first series of tests with one single double couple point source is deliberately simplistic. It is intended to serve as a reference for TR under near-ideal conditions. The TR method should be able to recover the point source, because otherwise there would be little hope for success in finitesource imaging.

The moment tensor point-source, with only $M_{x y}$ different from zero, is at $12.5 \mathrm{~km}$ depth. As source time function, we use a Gaussian wavelet with a dominant frequency of $1 \mathrm{~Hz}$. The wave field is computed for the 33 receivers shown in the left panel of Fig. 1. To illustrate the characteristics of the waveforms, a selection of N-S-component synthetic seismograms is shown in Fig. 2.

As suggested by Eq. (1), we monitor the time-reversed strain component $\epsilon_{x y}$. Snapshots of $\epsilon_{x y}$ at different times are shown at the point-source depth $(12.5 \mathrm{~km})$ in Fig. 3. The adjoint field starts to propagate from the stations with the largest epicentral distance and then focusses at the hypocentre as $t$ approaches 0 . Weaker or no focussing was observed for the other components of the strain tensor, as expected. While the focussing of $\epsilon_{x y}$ near the source can clearly be observed, $\left.\epsilon_{x y}\right|_{t=0}$ is still significantly different from zero in other regions of the model volume that are distant from the source. These "ghost waves" result from the imperfect reconstruction of the forward wave field by a finite number of irregularly spaced adjoint sources located at the surface. Depending on the particular setup, ghost waves may dominate the reverse field, thus, masking the focussing at the source location.

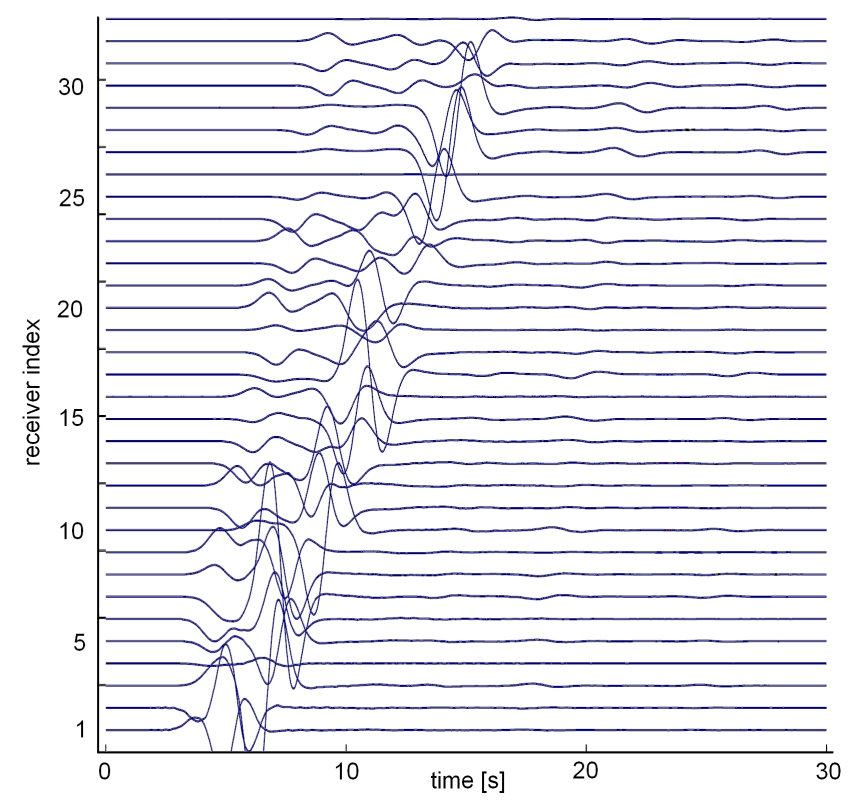

Fig. 2. N-S-component synthetic seismograms recorded at the 33 stations for a moment tensor point source with only $M_{x y} \neq 0$. The stations are sorted by distance to the epicentre and the traces are scaled to the maximum amplitude.

The influence of ghost waves can be reduced by using, for instance, the energy $E=\frac{1}{2} \mathbf{v}^{2}$ to image the source (Fig. 3, lower right). This leads to the suppression of contributions far from the source, but also to a less optimal focussing directly at the source location. In numerous experiments, a similar trade-off could be observed for other functionals of the time-reversed field, including the different components of the rotation vector $\nabla \times \mathbf{u}$ and the rotation energy $\frac{1}{2}(\nabla \times \mathbf{u})^{2}$. This suggests that time-reversal imaging always involves a compromise between the focussing at the source and the suppression of ghost waves.

Our test with a point source moment tensor demonstrates that focussing in space and time can indeed be observed, at least under the previously described circumstances. This result motivates the study of more complex scenarios. In the following, we focus our attention on the $x y$-component of the time-reversed strain field, $\epsilon_{x y}$. This restriction effectively corresponds to the injection of the prior information that the displacement on the infinitesimal or finite faults is a pure strike-slip.

\subsection{Multiple point sources}

Based on the encouraging results from the previous section, we add complexity to the source model and now consider three double couple point sources (only $M_{x y} \neq 0$ ) that are positioned along the fault of the SPICE Tottori benchmark (Fig. 1, left). The point sources have different initiation times 


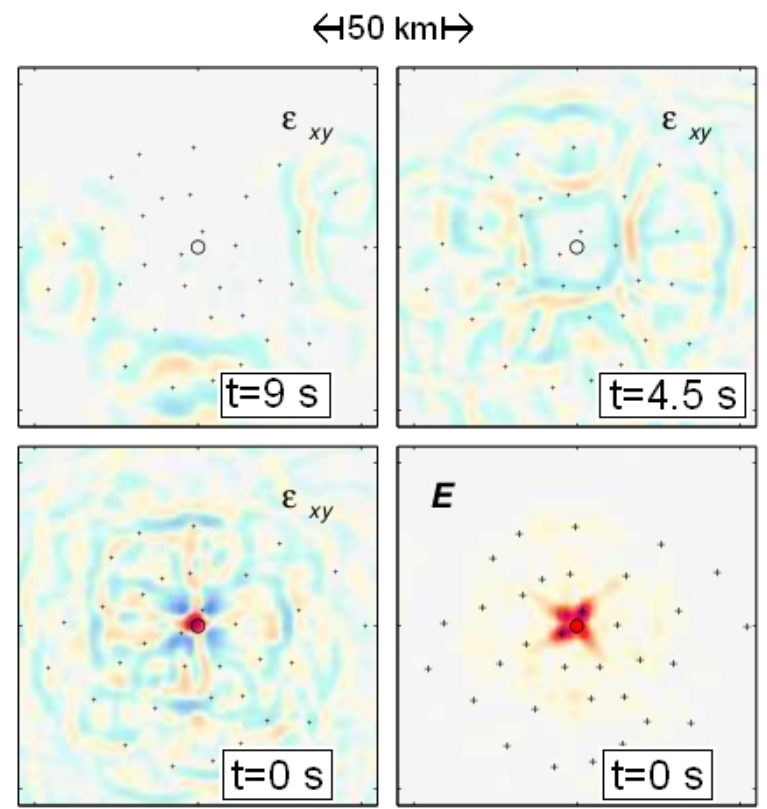

Fig. 3. Snapshots at the point-source depth $(12.5 \mathrm{~km})$ of the timereversed strain field $\epsilon_{x y}$ at different times, and the energy $\frac{1}{2} \mathbf{v}^{2}$ (lower right) at $t=0$.

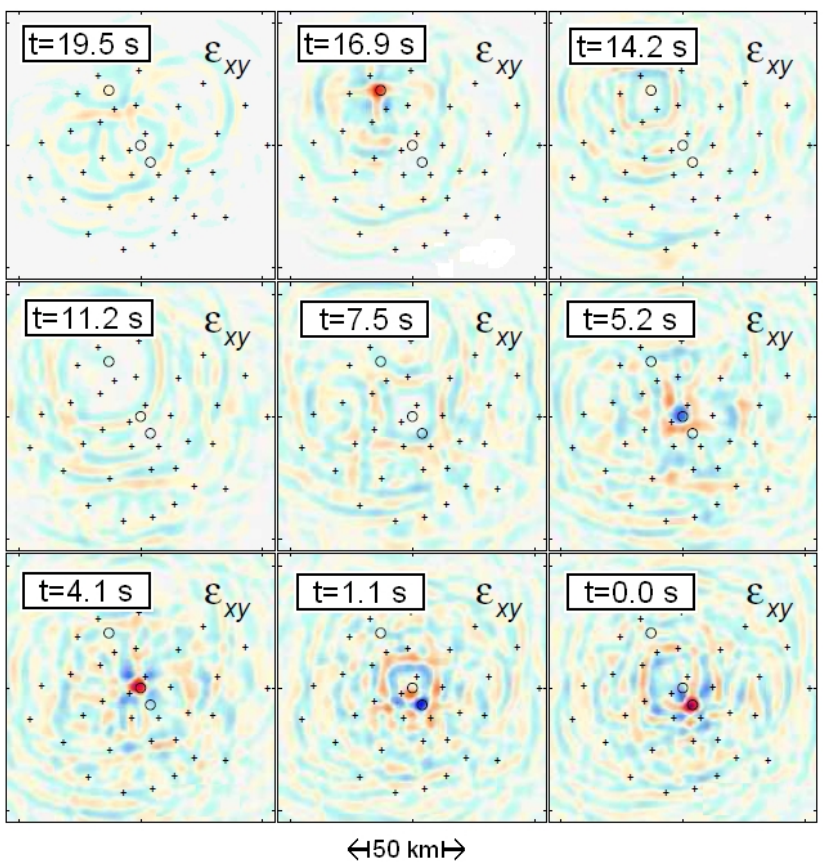

Fig. 4. Snapshots of the time-reversed strain field $\epsilon_{x y}$ at $12.5 \mathrm{~km}$ depth. Receiver and source locations are indicated by + and $\circ$, respectively. Focussing at all three source locations can be observed with an uncertainty of $\sim 5 \mathrm{~km}$ in space and $\sim 1 \mathrm{~s}$ in time. The observed hypothetical rupture velocity is $2 \pm 0.3 \mathrm{~km} \mathrm{~s}^{-1}$, compared to $2 \mathrm{~km} \mathrm{~s}^{-1}$ used to generate the forward wave field.
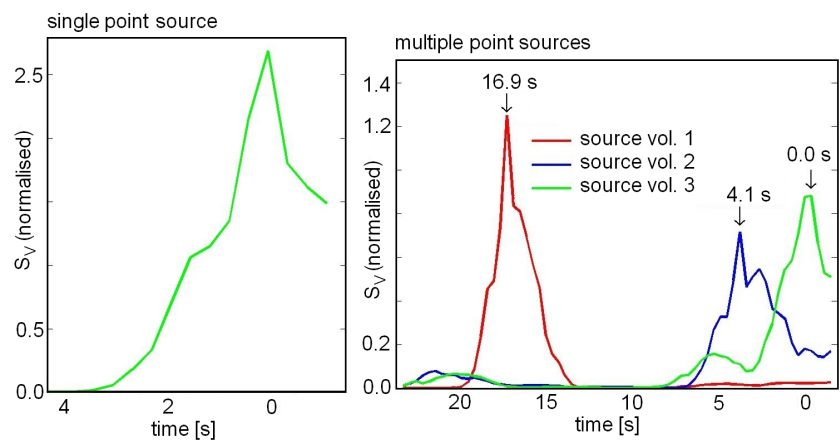

Fig. 5. Left: Time evolution of the normalised $S_{V}=\int_{V} \epsilon_{x y}^{2} d^{3} \mathbf{x}$ for the single point source experiment from section 3.1. A pronounced peak occurs at the focal time $t=0.0 \mathrm{~s}$. Right: The same as to the left, but for the multiple point source experiment from Sect. 3.2. Peaks can be observed at the focal times of the different point sources.

that correspond to a hypothetical rupture velocity of $2 \mathrm{~km} \mathrm{~s}^{-1}$ along the fault. The objective of this test is to reveal whether each of the three point sources can be resolved individually in both time and space.

Snapshots of the $x y$-component reverse strain, $\epsilon_{x y}$, are shown in Fig. 4. Circles mark the point source locations. Moving from the upper left to the lower right corner, we observe focussing at each of the three source locations around their respective initiation times of $16.9 \mathrm{~s}, 4.1 \mathrm{~s}$ and $0.0 \mathrm{~s}$, with an uncertainty of $\sim 1 \mathrm{~s}$. The width of the regions where focussing can be observed is $\sim 5 \mathrm{~km}$, which is close to the wavelength of the surface waves $(\sim 3 \mathrm{~km})$. From this we infer that the observed hypothetical rupture velocity is $2 \pm 0.3 \mathrm{kms}^{-1}$. We have, thus, obtained a first, and probably optimistic, estimate of the achievable space-time resolution in the subsequent finite-source imaging experiments.

\subsection{Quantitative assessment of focussing for point sources}

So far, a purely visual analysis of the time-reversed wave fields was sufficient to observe focussing. However, in anticipation of more complex finite-source scenarios, we examine the usefulness of a more quantitative criterion for the focal time within a pre-defined test volume: starting with the point source simulations we determine the quantity $S_{V}=\int_{V} \epsilon_{x y}^{2} d^{3} \mathbf{x}$ within a test volume $V$ around the source locations, and then consider the time when the maximum occurs as an estimate of the focal time. Since the wavelengths range between 4 and $20 \mathrm{~km}$, we let $V$ extend $10 \mathrm{~km}$ in all directions around the hypocentre location. As we seek a quantitative comparison of the focussing for various setups, we normalise $S_{V}$ by $S_{\otimes}=\int_{\otimes} \epsilon_{x y}^{2} d^{3} \mathbf{x}$, where $\otimes$ denotes the remaining model volume outside $V$.

Figure 5 shows the normalised $S_{V}$ for the single and multiple point source scenarios from Sects. 3.1 and 3.2, respectively. 


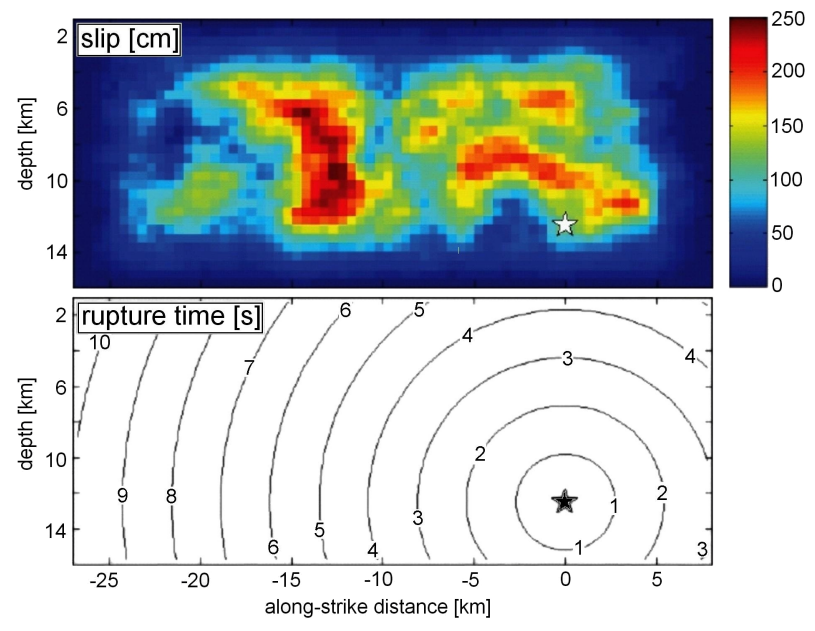

Fig. 6. Synthetic slip (top) and rupture time (bottom) distributions of the SPICE Tottori benchmark (Mai et al., 2007). Both the rupture speed and the rise time are constant at $v_{r}=2.7 \mathrm{~km} \mathrm{~s}^{-1}$ and $0.8 \mathrm{~s}$, respectively.

Distinct peaks at the expected source times are clearly visible in both cases. In the multiple point source experiment, we observe that the peaks for the first two sources (at $0.0 \mathrm{~s}$ and $4.1 \mathrm{~s}$ ) are comparatively low, probably due to their spatial proximity and overlapping test volumes.

We conclude that the analysis of $S_{V}$ is, at least for point sources, a useful diagnostic that allows us to estimate focal times and to compare the quality of focussing for different experimental setups.

Considering the multiple point source test successful, we now increase the complexity and make the transition to finite source models.

\section{Synthetic finite source simulations}

The SPICE kinematic source inversion blind test offers the opportunity to analyse the performance of TR finite source imaging. The blind test mimics the 2000 Tottori (Japan) earthquake that was recorded by a large number of strongmotion sensors. Figure 1 (left) shows the receiver configuration, the fault trace and the epicentre location. Synthetic seismograms for the 33 receivers are part of the benchmark package. They were generated by pure strike slip motion and with the slip and rupture time distributions shown in Fig. 6. The excited wave field has a maximum frequency of $3 \mathrm{~Hz}$.

Snapshots of the corresponding time-reversed strain component $\epsilon_{x y}$ are shown in the top panel of Fig. 4. In reverse time, the rupture propagates in NW-SE direction. However, a clear focus restricted to the fault plane cannot be observed - in contrast to our expectation. The wave field remains diffuse, compared to the previous point source simulations. A robust inference concerning the hypocentre location and the initiation time is not possible.
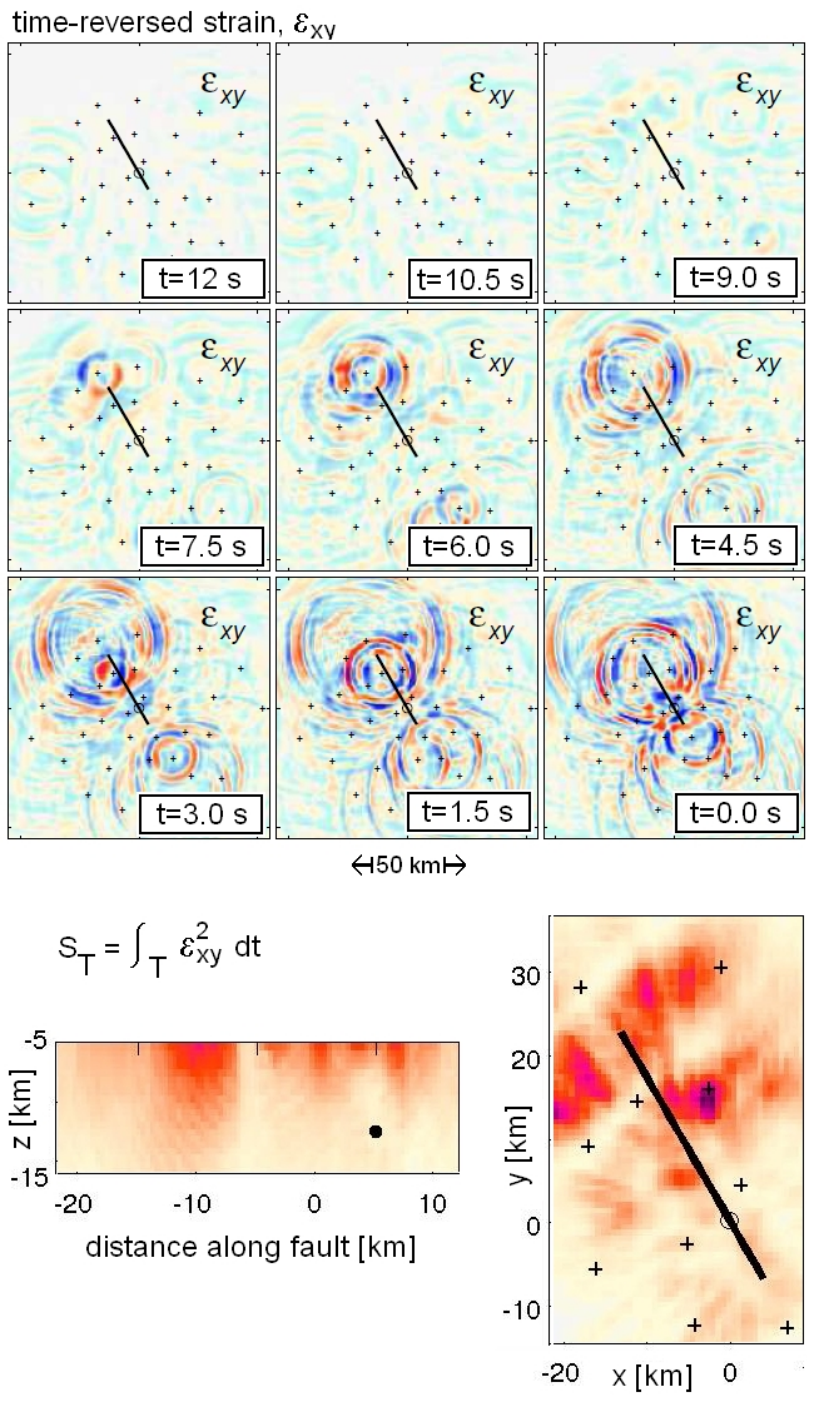

Fig. 7. Top: Snapshots of the time-reversed strain component $\epsilon_{x y}$ at $12.5 \mathrm{~km}$ depth. The fault trace is indicated by the black line. All snapshots are shown in the same amplitude range. Bottom: Cumulative squared strain $S_{T}=\int_{T} \epsilon_{x y}^{2} d t$ plotted on the fault plane (left) and integrated over depth (right).

In an attempt to facilitate the visual identification of both the fault and the rupture process, we analyse the cumulative squared strain $S_{T}=\int_{T} \epsilon_{x y}^{2} d t$. Based on physical intuition one would expect $S_{T}$ to be large only in those regions where significant strain occurs consistently over a longer period of time, i.e., along the fault. However, neither $S_{T}$ directly on the fault plane nor $S_{T}$ integrated over depth allow any meaningful inference concerning the location of the fault or the original slip distribution (see the bottom panels of Fig. 4). In fact, $S_{T}$ is largest near the surface, which reflects the dominance of surface waves in the time-reversed wave field. Moreover, $S_{T}$ on the fault plane reaches a local maximum where the original slip distribution (Fig. 6) is close to zero. The depthintegrated $S_{T}$ is largest far off the fault trace. 
Similar efforts to enhance the focussing on the fault by integrating, for instance, $\epsilon_{x y}$ or $\frac{1}{2} \mathbf{v}^{2}$ over time, did not lead to any significant improvements. We are, therefore, led to the early conclusion that no obvious functional of the timereversed field allows us to identify the fault plane or the slip distribution unambiguously. In what follows, we try to improve our results by (1) modifying the station distribution, and (2) weighting the adjoint sources.

\subsection{Modifications of the station distribution}

\subsubsection{Dense regular grid of stations}

The results from the previous section suggest that the number of stations and their spatial distribution provided insufficient information for the reconstruction of the original wave field. This motivates a synthetic test with a larger number of receivers (225 instead of 33) that are regularly spaced. While this scenario may be too optimistic in the near future, it provides valuable insight into TR finite source imaging under idealistic conditions.

For this experiment, we computed synthetic seismograms with the help of a Discontinuous Galerkin method (Käser and Dumbser, 2006) that allows us to model the discontinuous displacement on the fault with high accuracy. Snapshots of the resulting time-reversed strain component $\epsilon_{x y}$ are displayed in Fig. 8.

Compared to Fig. 4 (original station distribution), we observe a sharper peak. Most of the energy propagates along the fault plane and in a direction that is consistent with the rupture time distribution (6, bottom). However, the focus is still elongated perpendicular to the fault, which complicates its unambiguous identification. Any inference on the details of the original slip distribution (Fig. 6) remains clearly impossible.

To obtain more useful results, we again explored a variety of functionals of the time-reversed field, including the timeintegrated strain, the kinetic energy and the rotation amplitude. Neither of these functionals provided significant improvements, thus, confirming our earlier conclusion that the overall quality of the focussing is rather independent of the field used for imaging.

\subsection{Station arrays}

As an alternative to the previous densification of the receiver configuration, we investigate the installation of several small sub-arrays that are composed of four stations that form a $2 \mathrm{~km}$ by $2 \mathrm{~km}$ quadrangle. This geometry is intended to have a beam-forming effect that hopefully improves the focussing of the time-reversed field.

The corresponding time-reversed strain field $\epsilon_{x y}$ is shown in Fig. 9. The use of small sub-arrays clearly results in a more pronounced concentration of energy along the fault than with

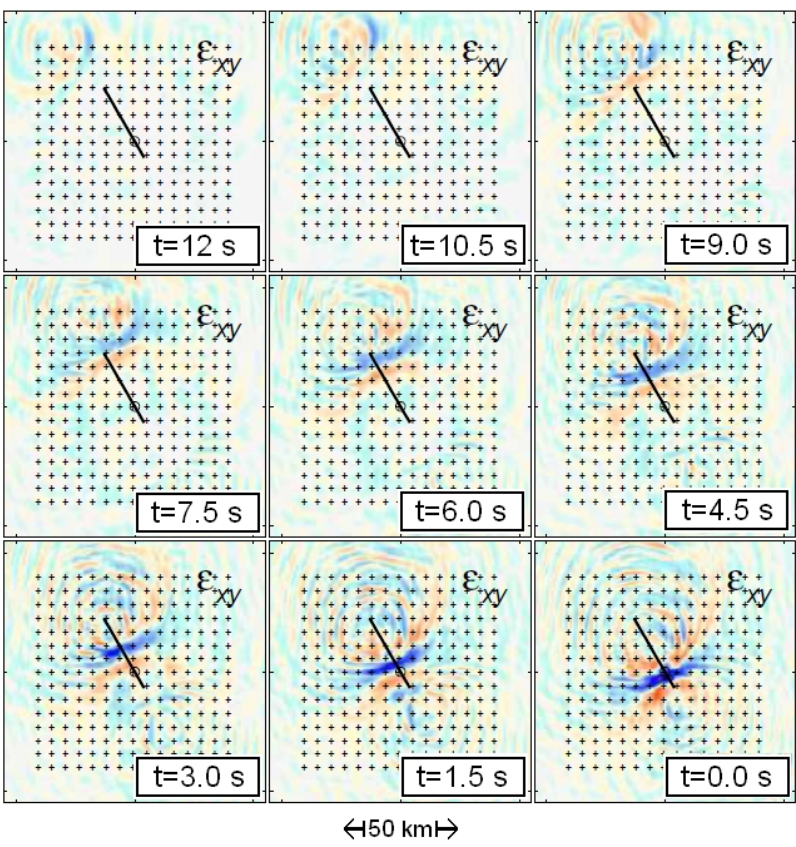

Fig. 8. Snapshots of the time-reversed strain component $\epsilon_{x y}$ at $12.5 \mathrm{~km}$ depth for the dense array of 225 regularly spaced receivers. The fault trace is indicated by the black line. All snapshots are shown in the same amplitude range.

the original station setup (Figs. 1 and 4). However, the problem of unambiguously identifying the fault itself remains unresolved also with this configuration. Again, the use of various functionals of the time-reversed field does not lead to significantly better results.

The previous experiments seem to imply that modifications of the receiver geometry are unlikely to improve the reconstruction of the original wave field to an extent that is sufficient to infer the slip distribution on the fault or even the fault itself.

\subsection{Weighting of adjoint sources}

A visual analysis of this failure (see Figs. 4 and 9) reveals that the highly unequal contributions from different receivers may be part of the problem. While receivers close to the fault dominate the time-reversed field due to the high amplitudes of the recorded waveforms, receivers at larger distances make only negligible contributions. This suggests that the reconstruction of the original wave field may be improved by assigning weights to the adjoint sources at position $\mathbf{x}^{r}$ that compensate for the geometric amplitude reduction with increasing propagation distance. In the following, we examine the effects of two different schemes where the weights are proportional to (1) the squared epicentral distance, and (2) the inverse energy of the recorded waveforms, i.e., $2 / \int \mathbf{v}\left(\mathbf{x}^{r}\right)^{2} d t$. It is important to note that the weighting scheme based on the distance from the epicentre corresponds to the incorporation 


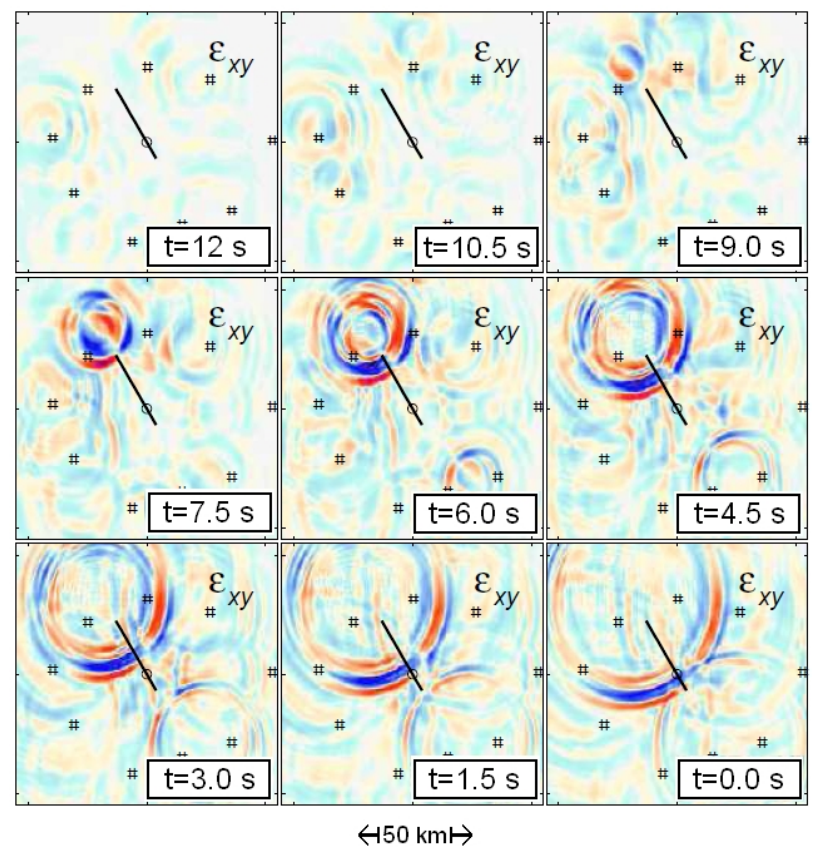

Fig. 9. Snapshots of the time-reversed strain component $\epsilon_{x y}$ at $12.5 \mathrm{~km}$ depth for the 9 sub-arrays composed of 4 receivers each. The fault trace is indicated by the black line. All snapshots are shown in the same amplitude range.

of prior information that may not be available in applications where the epicentral coordinates are among the unknowns.

Contrary to our expectations, the adjoint source weighting deteriorates the focussing of the time-reversed strain field, as can be seen in Fig. 10. The negative effect is strongest for the inverse energy weight. In both cases, neither the details of the rupture process nor the fault trace can be inferred from the images.

\section{Time-reverse imaging of the Tottori earthquake source}

One of the original motivations for this study was to use time reversal imaging in order to reveal the rupture details of the 2000 Tottori (Japan) earthquake. However, following the negative results of our finite-source synthetic experiments, we are forced to revise our ambitious goals, and to limit ourselves to the detection of the epicentre and the focal time.

For this real-data experiment, we use 111 surface recordings from the Japanese KiK-net (Fig. 11, http://www.kik. bosai.go.jp/), band-pass filtered between 0.03 and $0.5 \mathrm{~Hz}$ which is similar to the frequency range commonly used in kinematic source inversions. The dominant wavelength of the surface wave-dominated field is around $20 \mathrm{~km}$, which is close to the estimated fault length of $\sim 30 \mathrm{~km}$ (Semmane, 2005). We, therefore, expect to resolve only a point

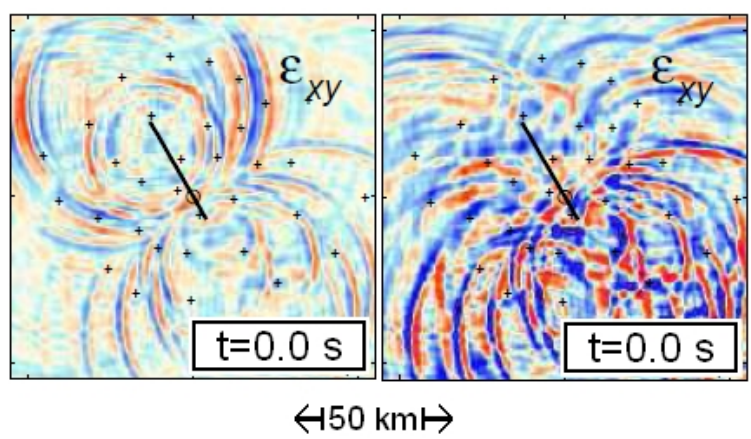

Fig. 10. Snapshots of the time-reversed strain component $\epsilon_{x y}$ at $12.5 \mathrm{~km}$ depth and $t=0$ for the weighted adjoint sources. Left: weight proportional to the squared epicentral distance. Right: weight proportional to the inverse energy $2 / \int \mathbf{v}\left(\mathbf{x}^{r}\right)^{2} d t$.

source. The amplitude at all adjoint sources was set equal to one to remove the strong influence of stations close to the source. For the time-reversed field wave propagation, we use the layered Earth model described in Semmane (2005). All data processing was done using ObsPy, a newly developed python-based toolbox for seismology (Beyreuther et al., 2010).

Figure 12 shows the propagation of the time-reversed strain component $\epsilon_{x y}$ from $t=30 \mathrm{~s}$ to $t=-30 \mathrm{~s}$. We observe clear focussing around the focal time and epicentre location as estimated by Semmane (2005). The uncertainty in time is $\sim 3 \mathrm{~s}$, and the uncertainty in space is around $50 \mathrm{~km}$, which is close to the dominant wavelength. The identification of the focal depth is not possible due to the comparatively long wavelengths and the presence of large-amplitude surface waves that mask the focussing of lower-amplitude body waves at greater depth. For negative times, i.e., prior to the initiation of the rupture, the time-reversed field propagates away from the epicentre, therefore, attesting to the appropriateness of the structural model. A very similar focussing and defocussing could be observed for the other strain components and various functionals of the time-reversed field.

To obtain a more quantitative estimate of the focal time, we proceed as in Sect. 3.3, where we computed the quantity $S_{V}=\int_{V} \epsilon_{x y} d^{3} \mathbf{x}$ for a volume $V$ surrounding the inferred point-source location. The time evolution of $S_{V}$, shown in Fig. 13, reveals a pronounced peak that serves as an estimate of the focal time. Our estimate positions the focal time at $+3 \mathrm{~s}$ relative to the initiation time of the rupture as inferred by Semmane (2005). This discrepancy is likely to be related to the inferred location of the Tottori hypocentre within an area of a small amount of final slip (e.g. Semmane, 2005; Piatanesi et al., 2007). Taking the corresponding rise times into account, this may explain a weak detectability by means of TR for the hypocentral parameters of the Tottori event. The peak in Fig. 13 is, therefore, likely to approximate the rupture time of the first large-slip region, or the centroid time 


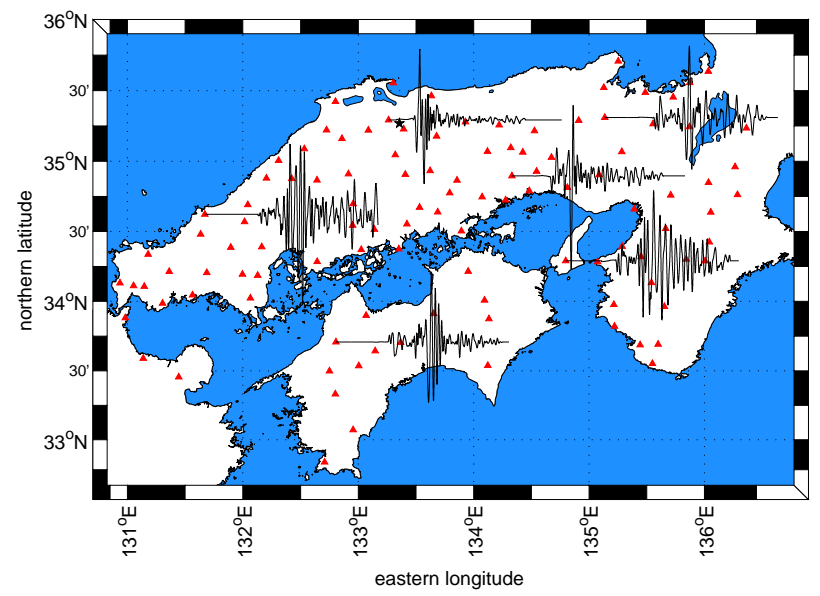

Fig. 11. Source-receiver geometry of the real-data TR experiment for the 2000 Tottori earthquake. Red triangles mark the positions of the 111 stations used in the experiment, and the black star indicates the epicentre as inferred by Semmane (2005). The seismograms shown are vertical component velocities in the chosen frequency band.

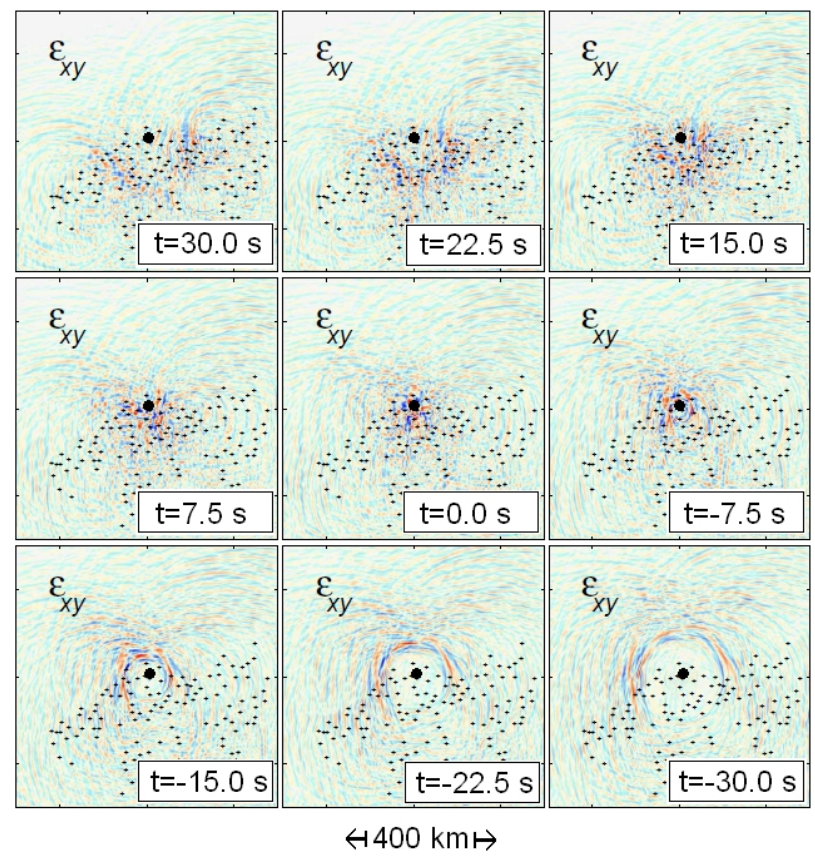

Fig. 12. Snapshots of the time-reversed strain component $\epsilon_{x y}$ at the surface for the Tottori data recorded at the 111 stations shown in Fig. 11. The coastlines are omitted to enhance the visibility of the time-reversed field. Estimates of both the focal time $(t=0 \mathrm{~s})$ and the epicentre location (black dot) are taken from Semmane (2005).

of the whole event (both at about $+4 \mathrm{~s}$, according to Semmane (2005) or Piatanesi et al. (2007) rather than the precise initiation time of the finite-size rupture.

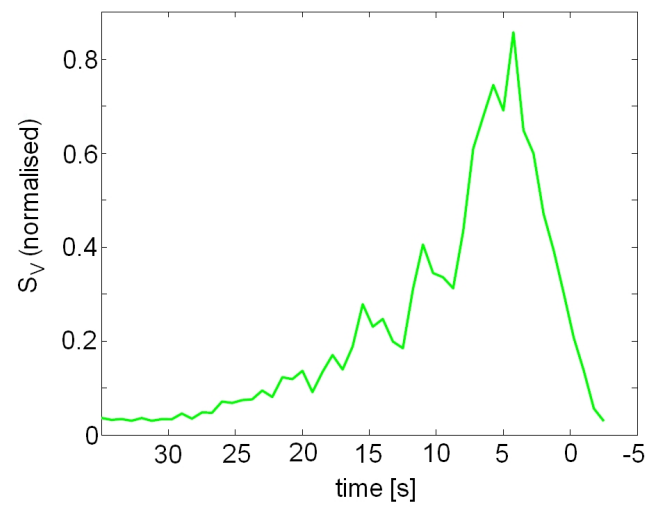

Fig. 13. Time evolution of the normalised $S_{V}=\int_{V} \epsilon_{x y}^{2} d^{3} \mathbf{x}$ for a volume $V$ that extends $20 \mathrm{~km}$ by $20 \mathrm{~km}$ around the epicentre as estimated from the time-reversal images from Fig. 12. The peak occurs at $+3 \mathrm{~s}$ relative to the focal time estimated by Semmane (2005) $(t=0)$.

\section{Discussion}

In the previous sections, we explored the potentials and limitations of the TR imaging of seismic sources on regional scales. For this we studied a variety of scenarios with both synthetic and real data.

The potential of the method clearly lies in the estimation of the location and the timing of point sources. In a series of synthetic experiments, we were able to observe the focussing of the time-reversed field in the vicinity of the original point source location and the original focal time. The uncertainties in the source location and time are governed by the frequency content and the receiver configuration. Our point source scenarios provide a proof of principle, but they are idealistic in the sense that we disregarded errors in the data and the assumed Earth model.

Our primary interest was in the detection of finite-rupture processes. Unfortunately, however, neither the rupture details nor the position of the fault itself could be inferred from the properties of the time-reversed wave field. To improve this result, we analysed various functionals of the wave field (strain, energy, rotations), modified the receiver geometry (densification, sub-arrays) and applied weights to the adjoint sources in order to compensate for geometric spreading. None of these strategies can be considered successful.

The reasons for this failure are manifold:

1. Incomplete information: Firstly and most importantly, the information recorded at the surface is plainly insufficient to reconstruct the original wave field with an accuracy that allows for the unambiguous identification of the rupture process. For instance, the body wave energy radiated downwards is entirely disregarded. This distinguishes TR on regional scales from TR on global scales where information is lost only through dissipation. 
2. Large-amplitude surface waves: Partly as a consequence of the previous item, the time-reversed field from stations that are distant from the fault is dominated by large-amplitude surface waves. The surface waves tend to mask the focussing of the lower-amplitude body waves that are primarily contributed by the stations closer to the fault. This effect results in a weak depth resolution, which means, in particular, that the focal depth can hardly be constrained.

3. The missing sink: An even more profound and general reason for failure is the incompleteness of the TR procedure. Our interest is in the seismic wave equation

$$
\rho \ddot{\boldsymbol{u}}(\boldsymbol{x}, t)-\nabla \cdot \boldsymbol{\sigma}(\boldsymbol{x}, t)=\boldsymbol{f}(\boldsymbol{x}, t)
$$

where $\boldsymbol{u}, \sigma$ and $\boldsymbol{f}$ denote the seismic displacement field, the stress tensor and an external force density. A complete time reversal of equation 2 would require the implementation of a sink $\boldsymbol{f}(\boldsymbol{x},-t)$ that acts as the counterpart of the source $\boldsymbol{f}(\boldsymbol{x}, t)$ in the forward direction, and that absorbs elastic energy so that the time-reversed field is zero for $t<0$. The sink, however, is disregarded simply because it is unknown. The missing sink poses a serious problem for finite-source inversions when fault segments are active at different times. The energy from segments that act late in the rupture process is not absorbed by the sink and, therefore, continues to propagate. The unabsorbed energy masks the focussing at the fault segments that act early in the rupture process. The immediate implication is that TR for finite sources is always dominated by those fault segments with large slip near the end of the rupture time.

4. Invisibility of small slip: A corollary of the previous item is that no information can be obtained about the rupture details on segments of the fault with small amount of final slip. This means, in particular, that the hypocentral parameters cannot be detected in those cases where the rupture initiation is associated with small slip.

5. Lack of prior information: The poor performance of TR finite-source imaging as compared to the classical kinematic source inversions is also due to the neglect of an apparently essential piece of prior information: The rupture occurs along a fault and is not diffusely distributed throughout the model volume.

6. Incomplete knowledge of the 3-D Earth structure: While excluded a priori in the synthetic experiments, inaccurate Earth models can prevent focussing in realdata applications. The focussing observed in our experiment with Tottori data suggests that the model is sufficient to explain at least the arrival times of the direct waves. However, the absence of horizontal het- erogeneities in the model does not allow for the correct back-propagation of scattered or even multiplescattered waves. This issue is closely related to

7. The insufficient complexity of 3-D Earth models that results either from the inherent smoothness of the Earth or the limited resolution of seismic tomography. The presence of strong multiple scattering is known to enhance focussing in laboratory experiments, but cannot be exploited in seismology where the knowledge about sub-wavelength heterogeneities is too inaccurate.

\section{Conclusions}

The principal conclusions to be drawn from our work are as follows: (1) Time-reversal imaging is well-suited to infer both the location and the timing of point sources. (2) Timereversal imaging in the used frequency range is not able to detect the details of finite rupture processes. Neither modifications of the receiver configuration (within reasonable bounds) nor the weighting of adjoint sources lead to sufficient improvements. (3) The dominant causes for this failure are the incomplete recordings of wave field information at the surface, the presence of large-amplitude surface waves that deteriorate the depth resolution, the missing sink that should absorb energy radiated during the later stages of the rupture process, the invisibility of small slip and the neglect of prior information.

While our experiments are certainly not exhaustive, they nevertheless suggest that the limitations of TR imaging start where the source stops being point-localised.

Acknowledgements. HI and SK gratefully acknowledge support through the Geophysics Group at Los Alamos National Labs for their research visits in 2008. We thank the Leibniz Rechenzentrum Munich for providing access to their supercomputer facilities and the IT support team (Jens Oeser) at the Geophysics Section of the Department of Earth Sciences at LMU Munich. We are also grateful to Martin Mai for giving us access to the SPICE dataset. We are grateful to J.-P. Montagner and B. Artman whose comments improved the manuscript. This work would not have been possible without the support of the European Commission in connection with the Research and Training Network SPICE (spice-rtn.org) and the Initial Training Network QUEST (quest-itn.org).

Edited by: R. Carbonell 


\section{References}

Allmann, B. P. and Shearer, P. M.: A high-frequency secondary event during the 2004 Parkfield earthquake., Science (New York, N.Y.), 318, 1279-83, doi:10.1126/science.1146537, http://www. ncbi.nlm.nih.gov/pubmed/18033878, 2007.

Artman, B., Podladtchikov, I., and Witten, B.: Source location using time-reverse imaging, Geophys. Prospect., 58, 861-873, doi:10.1111/j.1365-2478.2010.00911.x, 2010.

Beyreuther, M., Barsch, R., Krischer, L., Megies, T., Behr, Y., and Wassermann, J.: ObsPy: A Python Toolbox for Seismology, Seismol. Res. Lett., 81, 530-533, doi:10.1785/gssrl.81.3.530, 2010.

Bunge, H.-P., Hagelberg, C. R., and Travis, B. J.: Mantle circulation models with variational data assimilation: inferring past mantle flow and structure from plate motion histories and seismic tomography, Geophys. J. Int., 152, 280-301, doi:10.1046/j.1365246X.2003.01823.x, 2003.

Cesca, S., Heimann, S., Stammler, K., and Dahm, T.: Automated procedure for point and kinematic source inversion at regional distances, J. Geophys. Res., 115, B06304, doi:10.1029/2009JB006450, 2010.

Chakroun, N., Fink, M., and Wu, F.: Time reversal processing in ultrasonic nondestructive testing, IEEE T. Ultrason. Ferr., 42, 1087-1098, doi:10.1109/58.476552, 1995.

Cotton, F. and Campillo, M.: Frequency domain inversion of strong motions: Application to the 1992 Landers earthquake, J. Geophys. Res., 100, 3961-3975, 1995.

Derode, A., Roux, P., and Fink, M.: Robust Acoustic Time Reversal with High-Order Multiple Scattering, Phys. Rev. Lett., 75, 42064209, doi:10.1103/PhysRevLett.75.4206, 1995.

Edelmann, G., Akal, T., Hodgkiss, W., and Kuperman, W.: An initial demonstration of underwater acoustic communication using time reversal, IEEE Journal of Oceanic Engineering, 27, 602609, doi:10.1109/JOE.2002.1040942, 2002.

Fichtner, A.: Full seismic waveform modelling and inversion, Springer, Heidelberg, 2010.

Fichtner, A. and Igel, H.: Efficient numerical surface wave propagation through the optimization of discrete crustal modelsa technique based on non-linear dispersion curve matching (DCM), Geophys. J. Int., 173, 519-533, doi:10.1111/j.1365246X.2008.03746.x, 2008.

Fichtner, A., Bunge, H., and Igel, H.: The adjoint method in seismologyI. Theory, Phys. Earth Planet. In., 157, 86-104, doi:10.1016/j.pepi.2006.03.016, 2006.

Fichtner, A., Igel, H., Bunge, H.-P., and Kennett, B. L. N.: Simulation and Inversion of Seismic Wave Propagation on Continental Scales Based on a Spectral-Element Method, Journal of Numerical Analysis, Industrial and Applied Mathematics, 4, 11-22, 2009a.

Fichtner, A., Kennett, B. L. N., Igel, H., and Bunge, H.-P.: Full seismic waveform tomography for upper-mantle structure in the Australasian region using adjoint methods, Geophys. J. Int., 179, 1703-1725, doi:10.1111/j.1365-246X.2009.04368.x, 2009b.

Fink, M.: Time reversal in acoustics, Contemp. Phys., 37, 95-109, 1996.

Fink, M.: Time reversed acoustics, Phys. Today, 50, 34-40, 1997.

Fink, M. and Tanter, M.: Multiwave imaging and super resolution, Physics Today, 63, 28-33, 2010.
Gajewski, D. and Tessmer, E.: Reverse modelling for seismic event characterization, Geophys. J. Int., 163, 276-284 doi:10.1111/j.1365-246X.2005.02,732.x, 2005.

Hjörleifsdóttir, V.: Earthquake source characterization using 3D Numerical Modeling, Ph.D. thesis, California Institute of Technology, Pasadena, California, USA, 2007.

Ishii, M., Shearer, P. M., Houston, H., and Vidale, J. E.: Extent, duration and speed of the 2004 Sumatra-Andaman earthquake imaged by the Hi-Net array., Nature, 435, 933-6, doi:10.1038/nature03675, 2005.

Käser, M. and Dumbser, M.: An arbitrary high-order discontinuous Galerkin method for elastic waves on unstructured meshes - I. The two-dimensional isotropic case with external source terms, Geophys. J. Int., 166(2), 855-877, doi:10.1111/j.1365246X.2006.03,051.x, 2006.

Kennett, B. L. N.: A new way to estimate seismic source parameters, Nature,f 302, 659-660, 1983.

Larmat, C., Montagner, J.-P., Fink, M., Capdeville, Y., Tourin, A., and Clévédé, E.: Time-reversal imaging of seismic sources and application to the great Sumatra earthquake, Geophys. Res. Lett., 33, L19312, doi:10.1029/2006GL026336, 2006.

Larmat, C. S., Guyer, R. A., and Johnson, P. A.: Tremor source location using time reversal: Selecting the appropriate imaging field, Geophys. Res. Lett., 36, L22304, doi:10.1029/2009GL040099, 2009.

Mai, P., Burjanek, J., Delouis, B., Festa, G., Francois-Holden, C., Monelli, D., Uchide, T., and Zahradnik, J.: Earthquake Source Inversion Blindtest: Initial Results and Further Developments, AGU Fall Meeting San Francisco, 2007.

McMechan, G. A.: Determination of source parameters by wavefield extrapolation, Geophys. J. R. astr. SOC., 71, 613-628, doi:10.1111/j.1365-246X.1982.tb02788.x, 1982.

McMechan, G. A., Luetgert, J. H., and Mooney, W. D.: Imaging of earthquake sources in Long Valley Caldera, California, 1983, Bull. Seismol. Soc. Am., 75, 1005-1020, 1985.

O’Brien, G. S., Lokmer, I., De Barros, L., Bean, C. J., Saccorotti, G., Metaxian, J.-P., and Patane, D.: Time reverse location of seismic long-period events recorded on Mt Etna, Geophys. J. Int., 184, 452-462, doi:10.1111/j.1365-246X.2010.04851.x, 2011.

Parvulescu, A. and Clay, C. S.: Reproducibility of signal transmissions in the ocean, Radio Electron. Eng., 29, 223-228, doi:10.1049/ree.1965.0047, 1965.

Piatanesi, A., Cirella, A., Spudich, P., and Cocco, M.: A global search inversion for earthquake kinematic rupture history: Application to the 2000 western Tottori, Japan earthquake, J. Geophys. Res., 112, B07314, doi:10.1029/2006JB004821, 2007.

Rietbrock, A. and Scherbaum, F.: Acoustic imaging of earthquake sources from the Chalfant Valley, 1986, aftershock series, Geophys. J. Int., 119, 260-268, doi:10.1111/j.1365246X.1994.tb00926.x, 1994.

Semmane, F.: The 2000 Tottori earthquake: A shallow earthquake with no surface rupture and slip properties controlled by depth, J. Geophys. Res., 110, B03306, doi:10.1029/2004JB003194, 2005.

Steiner, B., Saenger, E. H., and Schmalholz, S. M.: Timereverse modeling of microtremors: Application to hydrocarbon reservoir localization, Geophys. Res. Lett., 35, L03307, doi:10.1029/2007GL032097, 2008.

Sun, N.-Z.: Inverse problems in ground water modelling, Kluwer Academic Publishers, 1994. 
Sutin, A. M., TenCate, J. A., and Johnson, P. A.: Single-channel time reversal in elastic solids, The Journal of the Acoustical Society of America, 116, 2779, doi:10.1121/1.1802676, 2004.

Talagrand, O. and Courtier, P.: Variational Assimilation of Meteorological Observations With the Adjoint Vorticity Equation. I: Theory, Q. J. Roy. Meteor. Soc., 113, 1311-1328, doi:10.1002/qj.49711347812, 2007.
Tarantola, A.: Theoretical background for the inversion of seismic waveforms including elasticity and attenuation, Pure Appl. Geophys., 128, 365-399, doi:10.1007/BF01772605, 1988.

Tromp, J., Tape, C., and Liu, Q.: Seismic tomography, adjoint methods, time reversal and banana-doughnut kernels, Geophys. J. Int., 160, 195-216, doi:10.1111/j.1365-246X.2004.02453.x, 2004. 